\title{
Decisions Relating to Cardiopulmonary Resuscitation: a joint statement from the British Medical Association, the Resuscitation Council (UK) and the Royal College of Nursing
}

\section{Summary}

Principles

- Timely support for patients and people close to them, and effective, sensitive communication are essential.

- Decisions must be based on the individual patient's circumstances and reviewed regularly.

- Sensitive advance discussion should always be encouraged, but not forced.

- Information about CPR and the chances of a successful outcome needs to be realistic.

\section{Practical matters}

- Information about CPR policies should be displayed for patients and staff.

- Leaflets should be available for patients and people close to them explaining about CPR, how decisions are made and their involvement in decisions.

- Decisions about attempting CPR must be communicated effectively to relevant health professionals.

\section{In emergencies}

- If no advance decision has been made or is known, CPR should be attempted unless:

- the patient has refused CPR;

- the patient is clearly in the terminal phase of illness; or

- the burdens of the treatment outweigh the benefits.

\section{Advance decision making}

- Competent patients should be involved in discussions about attempting CPR unless they indicate that they do not want to be.

- Where patients lack competence to participate, people close to them can be helpful in reflecting their views.

\section{Legal issues}

- Patients' rights under the Human Rights Act must be taken into account in decision making.

- Neither patients nor relatives can demand treatment which the health care team judges to be inappropriate, but all efforts will be made to accommodate wishes and preferences.

- In England, Wales and Northern Ireland relatives and people close to the patient are not entitled in law to take health care decisions for the patient.

- In Scotland, adults may appoint a health care proxy to give consent to medical treatment.

- Health professionals need to be aware of the law in relation to decision making for children and young people. 


\section{Introduction}

\section{Why policies are needed}

Cardiopulmonary resuscitation (CPR) can be attempted on any person whose cardiac or respiratory functions cease. Failure of these functions is part of dying and thus CPR can theoretically be attempted on every individual prior to death. But because for every person there comes a time when death is inevitable, it is essential to identify patients for whom cardiopulmonary arrest represents a terminal event in their illness and in whom attempted CPR is inappropriate. It is also essential to identify those patients who do not want CPR to be attempted and who competently refuse it.

All establishments that face decisions about attempting CPR, including hospitals, general practices, residential care homes and ambulance services, should have in place a policy about resuscitation attempts. NHS Trust chief executives are required to ensure that policies are in place that respect patients' rights, are understood by all relevant staff and accessible to those who need them. The process by which policies are drawn up is a matter to be decided locally. Some establishments may want to involve groups of health professionals and patient representatives.

\section{The purpose of these guidelines}

Health professionals are aware that decisions about attempting resuscitation raise very sensitive and potentially distressing issues for the patient and people emotionally close to the patient. The guidelines that follow identify the key ethical and legal issues that should underpin all these decisions. These basic principles are the same for all patients, in all settings, but because a range of clinical and personal matters has to be taken into account in each case, the decision arrived at in the care of one patient may be inappropriate in a superficially similar case.

These guidelines do not seek to address the complex clinical considerations health teams face, and do not cover the practical issues which vary from setting to setting. The way decisions are made and the factors that are important in accident and emergency departments, for example, are not the same as in residential nursing homes for older people, in intensive treatment units, or for people caring for children or people with learning disabilities. The guidelines do not distinguish between basic and advanced CPR since the underlying ethical and legal principles about how decisions should be reached are the same. Local policy makers may find it helpful, however, to tailor policies to their own particular setting, to ensure they are relevant to the type of patients being cared for and take account of what facilities are available. These guidelines should, therefore, be viewed as a framework providing basic principles within which local policies on CPR attempts may be formulated. Local policies may also encompass more detailed guidance with more specific information about individuals' responsibilities than can be provided here.
Further assistance for doctors and nurses regarding particular problem areas can be sought from their respective professional organisations. Details of some additional published guidance is given in section 18 .

\section{Legal issues}

Policies about decisions relating to $\mathrm{CPR}$ require review following the implementation of the Human Rights Act 1998 on 2 October 2000. This Act incorporates the bulk of the rights set out in the European Convention on Human Rights into UK law. In order to meet their obligations under the Act, health professionals must be able to show that their decisions are compatible with the human rights set out in the Articles of the Convention. Provisions particularly relevant to decisions about attempted CPR include the right to life (Article 2), to be free from inhuman or degrading treatment (Article 3), to respect for privacy and family life (Article 8), to freedom of expression, which includes the right to hold opinions and to receive information (Article 10) and to be free from discriminatory practices in respect of these rights (Article 14). The spirit of the Act, which aims to promote human dignity and transparent decision making, is reflected in these ethical guidelines. If there is any doubt about the legality of an advance decision about attempting resuscitation, legal advice should be sought.

\section{Guidelines}

\section{The goal of medicine}

The primary goal of medical treatment is to benefit patients, by restoring or maintaining their health as far as possible, thereby maximising benefit and minimising harm. If treatment fails, or ceases, to give a net benefit to the patient, or if an adult patient has competently refused the treatment, this goal cannot be realised and the justification for providing the treatment is removed.

Prolonging a patient's life usually provides a health benefit to that patient. Nevertheless, it is not an appropriate goal of medicine to prolong life at all costs with no regard to its quality or the burdens of treatment on the patient. Some mentally competent patients decide that the stage has been reached beyond which, for them, continued treatment aimed at prolonging life, although possible, would be inappropriate. Some identify a point in the future after which they do not want treatment, and refuse further interventions in an advance directive (see section 9). If these decisions are informed and not coerced, they must be respected. Where patients have no advance directive and are not competent, decisions about whether to provide treatment must be taken in a way that reflects their best interests, an assessment of which includes their clinical interests, plus any current and previously expressed wishes and preferences. Where there is no information about their wishes or preferences, decisions must be consistent with and not contrary 
312 Clinical ethics: Decisions Relating to Cardiopulmonary Resuscitation: a joint statement from the British Medical Association, the Resuscitation Council (UK) and the Royal College of Nursing

to their interests or rights. The emphasis on the individual's interests means that it is important that resuscitation is discussed sensitively with competent patients and people close to incompetent patients. This can help people to understand why treatment is given and why, in some circumstances, it may be unable to provide any benefit.

\section{Presumption in favour of attempting resuscitation}

Where no explicit advance decision has been made about the appropriateness or otherwise of attempting resuscitation prior to a patient suffering cardiac or respiratory arrest, and the express wishes of the patient are unknown and cannot be ascertained, there should be a presumption that health professionals will make all reasonable efforts to attempt to revive the patient. Anyone attempting CPR in such circumstances should be supported by their senior medical and nursing colleagues. Although this is the general assumption, it is unlikely to be considered reasonable to attempt to resuscitate a patient who is in the terminal phase of illness or for whom the burdens of the treatment clearly outweigh the potential benefits.

Local policies in hospitals should particularly include instructions for junior doctors on what to do in an emergency situation where there is no consultant immediately available.

\section{Essential aspects of decision making}

Each case involves an individual patient with his or her own particular circumstances and it is important to ensure that these circumstances are central to each decision rather than applying the same decision to whole categories of patients.

Ideally, decisions about whether to attempt to resuscitate a particular patient are made in advance as part of overall care planning for that patient and, as such, are discussed with the patient along with other aspects of future care. An advance decision that CPR will not be attempted (a "do not attempt resuscitation", or "DNAR" order) should be made only after the appropriate consultation and consideration of all relevant aspects of the patient's condition. These include:

- the likely clinical outcome, including the likelihood of successfully restarting the patient's heart and breathing, and the overall benefit achieved from a successful resuscitation;

- the patient's known, or ascertainable, wishes; and

- the patient's human rights, including the right to life and the right to be free from degrading treatment.

The views of all members of the medical and nursing team, including those involved in the patient's primary and secondary care and, with due regard to patient confidentiality, people close to the patient, are valuable in forming the decision. Once made, al decisions must be communicated effectively to the relevant health professionals. Local policies should specify how, and by whom, decisions will be communicated.

\section{Information to patients}

Written information about resuscitation policies should be included in the general literature provided to patients about health care establishments, including Trusts, hospitals, general practices and residential care facilities. Such information should be readily available to all patients and to people close to the patient including relatives and partners. Its purpose is to de-mystify the process by which decisions are made and to explain what facilities are available. Patients should be encouraged to see such information as a routine part of advance care planning to cover all contingencies rather than an intimation of a particular risk to themselves. Information should reassure patients of their part in decision making and should make clear that for most patients the question may not arise. Nevertheless, all patients and those close to them can ask for time to be set aside to discuss the issues if they feel they will be relevant to them. Local policies may include mention of how such non-urgent discussions can best be accommodated, and where people can go for further advice and information.

\section{Competent adults}

\subsection{Discussion}

Discussions of the advisability or otherwise of attempting CPR are highly sensitive and complex and should be undertaken by senior, experienced members of the medical team who have received appropriate training in communication skills. Support should come from similarly trained senior nursing colleagues.

People have ethical and legal rights to be involved in decisions that relate to them. Because patients' own views about the level of burden or risk they consider acceptable carry considerable weight in deciding whether treatment is given, it follows that decisions about whether the likely benefits from successful CPR outweigh the burdens should be discussed with competent patients. Thus where competent patients are at foreseeable risk of cardiopulmonary arrest, or have a terminal illness, there should be sensitive exploration of their wishes regarding resuscitation. This will normally arise as part of general discussions about that patient's care. Information should not be forced on unwilling recipients, however, and if patients indicate that they do not wish to discuss resuscitation this should be respected. Competent patients should understand that there are opportunities to talk about attempting CPR, but should not be forced to discuss the issue if they do not want to. Where a DNAR order is made and there has been no discussion with the patient because he or she has indicated a clear desire to avoid such discussion, this must be documented in the health records and the reasons given. As with any other aspect of care, health professionals must be able to justify their actions.

For the vast majority of patients receiving care in hospital the likelihood of cardiopulmonary arrest is small and no advance decision is made. If 
cardiopulmonary arrest does occur, resuscitation is attempted in accordance with the advice in section 2 of these guidelines. There is no ethical or legal requirement to discuss every possible eventuality with all patients, although if patients for whom cardiopulmonary arrest is not a foreseeable likelihood do want to discuss resuscitation, the health team must be willing to do this and to answer any questions honestly.

Whether successful CPR is able to provide any benefit to the patient is the key question in deciding about whether it should be attempted. Health professionals should ensure that their recommendations about whether CPR should be attempted are based on the best available data and, if it can be achieved, consensus within the team. They should also sensitively portray to patients the inherent uncertainty in this type of clinical decision. It is problematic for health professionals that many patients have unrealistic expectations about the potential benefits of CPR and lack detailed information about what is involved. The picture gained from the media (television drama for example) seldom reflects a realistic view of the success rate, nor the physical nature of a prolonged resuscitation attempt. While health professionals understandably are reluctant to alarm patients or deter them from treatment which can be life-saving, it is important that people involved in decisions are aware of what is involved and of the variables which affect success.

Any discussions about whether to attempt CPR, and any anticipatory decisions, should be documented, signed and dated in the patient's record.

\subsection{Refusal of a DNAR order and patient requests for attempted CPR}

Some patients may ask for CPR to be attempted, even if the clinical evidence suggests that in their case it will not effectively restart the heart and breathing or that it cannot provide any overall benefit. Sensitive efforts should be made without alarming the patient to convey a realistic view of the procedure and its likely success. Discussion should aim at securing an understanding and acceptance of the clinical judgement. If patients still ask that no DNAR order be made, this should be respected. If a situation in which CPR is a practical option for such patients then arises, the decision must be made in accordance with the advice in section 2 of these guidelines. Doctors cannot be required to give treatment contrary to their clinical judgement, but should, whenever possible, respect patients' wishes to receive treatment which carries only a very small chance of success or benefit.

\section{Incapacitated adults}

People close to patients often have the perception that they have the final say about whether CPR should be attempted, yet in England, Wales and Northern Ireland, no person is legally entitled to give consent to medical treatment on behalf of an adult who lacks decision making capacity. Doctors have authority to act in their patients' best interests where consent is unavailable. Unless to do so would be contrary to the patient's interests, people close to the patient should be kept informed about the patient's health and be involved in decision making in order to reflect the patient's views and preferences. It should be made clear that their role is not to take decisions on behalf of the patient. Relatives and others close to the patient should be assured that their views on what the patient would want will be taken into account in decision making but they cannot insist on treatment or non-treatment (see also section 8).

Additionally in Scotland, the Adults with Incapacity (Scotland) Act allows people over 16 to appoint a proxy decision maker who has the legal power to give consent to medical treatment when the patient loses capacity. Unless to do so is unreasonable or impracticable, the proxy must be consulted about treatment decisions. Proxy decision makers cannot demand treatment which is judged to be against the patient's interests. The Act also requires doctors to take account, so far as is reasonable and practicable, of the views of the patient's nearest relative and his or her primary carer.

\section{Children and young people}

It is recognised widely that medical decisions relating to children and young people ideally should be taken within a supportive partnership involving patients, their families and the health care team. The views of children and young people must be taken into consideration in decisions about attempting CPR.

Competent young people are entitled to give consent to medical treatment, and where they lack competence it is generally their parents who make decisions on their behalf. In England, Wales and Northern Ireland, refusal of treatment by competent young people is not necessarily binding upon doctors since the courts have ruled that consent from people with parental responsibility, or the court, still allows doctors to provide treatment. The Human Rights Act may have implications for such situations, but at the time of writing these guidelines no relevant legal cases had arisen to clarify the legal position. Where a competent young person refuses treatment, the harm caused by violating the young person's choice must be balanced against the harm caused by failing to treat. In Scotland, it is likely that neither parents nor the courts are entitled to override a competent young patient's decision.

Usually agreement will be reached about whether CPR should be attempted if the patient suffers respiratory or cardiac failure. If disagreement persists despite attempts to reach agreement, legal advice should be sought. Parents cannot require doctors to provide treatment contrary to their professional judgement, but doctors will try to accommodate parents' wishes as far as is compatible with protecting the child's interests.

\section{Involving people close to the patient}

Despite feeling that they are the natural decision makers, people close to patients frequently report 
314 Clinical ethics: Decisions Relating to Cardiopulmonary Resuscitation: a joint statement from the British Medical Association, the Resuscitation Council (UK) and the Royal College of Nursing

feeling excluded from a range of decision making. Even where their views have no legal status in terms of actual decision making, it is good practice to involve people close to patients in decisions. If the patient is competent, his or her agreement should be sought. It may also be helpful to ask competent patients who they want, or do not want, to be generally involved in decision making if they become incapacitated. Refusal by a competent patient to allow information to be disclosed to family or friends should be respected. Where an incompetent patient's views on involving family and friends are not known, doctors may disclose confidential information to people close to the patient where this is necessary to discuss the patient's care and not contrary to the patient's interests. It is important to be clear that the information sought from people close to patients is to help ascertain what the patient would have wanted in these circumstances, as opposed to what those consulted would like for the patient or what they would want for themselves if they were in the same situation.

Health professionals should be aware that the requirement to respect family life and impart information are important human rights considerations. Where patients have become incapacitated, relatives can provide important information to help ascertain the patient's prior views about treatment. As noted above, these need to be factored into any decision but may not ultimately be determinative. The European Court of Human Rights has taken the view that parents have the right under Article 8 of the European Convention to be involved in important decisions concerning their children. By analogy, it is arguable that excluding the family of incompetent patients also breaches this right unless the patient previously instructed it.

\section{Refusal of treatment}

Resuscitation must not be attempted if CPR is contrary to the recorded, sustained wishes of an adult who was mentally competent and aware of the implications at the time of making that advance decision. Refusal of treatment by children and young people is dealt with in section 7 .

It is well established in law and ethics that competent adults have the right to refuse any medical treatment, even if that refusal results in their death. Such a patient's informed and competently made refusal which relates to the circumstances which have arisen is legally binding upon doctors. Some patients choose to express their wishes in a written document, an advance directive or "living will" but it is not necessary for refusal to be in writing in order to be valid. People often discuss their wishes with a GP or another health professional who records the discussion in the patient's notes. Where patients express a clear and consistent refusal, this is likely to have the same status as a written advance directive.

Patients are not obliged to justify their decisions, but need to ensure that the health team is aware of them if they are to be implemented. Health professionals usually wish to discuss the implications of the refusal with patients in order to ensure that the decision is based on accurate information and not on a misunderstanding, but must take care not to pressure patients into accepting treatment they do not want.

Effective communication of a refusal of treatment is essential. This is discussed in section 11 .

\section{When is it appropriate to consider making a DNAR order?}

This section outlines situations in which it is appropriate to consider making a DNAR order. It does not define the circumstances in which patients must and must not be resuscitated. Although health professionals are familiar with the concept of not providing "futile" treatment, precise definitions of what constitutes "futility" vary. Therefore these guidelines avoid the term and attempt to specify, in each instance, the type of factors that are important in decisions about whether attempting CPR is appropriate. As has been emphasised throughout, the appropriate course of action may be very different in apparently similar circumstances, because the wishes and preferences of the patient are an essential element of the decision.

\subsection{Where attempting CPR will not restart the patient's heart and breathing}

If the health care team is as certain as it can be that attempting CPR would not restart the patient's heart and breathing, the patient cannot gain any clinical benefit from an attempt. Consensus within the team about likely clinical outcome should be the aim, and decision making must be based on clinical assessment of the patient's condition and up-to-date clinical guidelines.

\subsection{Where there is no benefit in restarting the patient's heart and breathing}

Although in most cases there is benefit gained when a patient's heart and breathing are successfully restarted following cardiopulmonary arrest, this is not true in all cases. No benefit is gained if only a very brief extension of life can be achieved and the patient's co-morbidity is such that imminent death cannot be averted. Similarly no benefit is gained by the patient if he or she will never have awareness or the ability to interact and is therefore unable to experience benefit.

\subsection{Where the expected benefit is outweighed by the burdens}

Where CPR may be successful in restarting the patient's heart and breathing, and thus prolong the patient's life, the benefits to be gained from the prolongation of life must be weighed against the burdens to the patient of the treatment.

There are many circumstances in which it is justified to attempt to prolong life by treatment which carries side effects, burdens and risks. Attempted CPR carries a risk of significant side effects (such as 
sternal fracture, rib fracture and splenic rupture) and most patients require either coronary care or intensive care treatment in the post resuscitation period. If there is delay between cardiopulmonary arrest and the resuscitation attempt, there is a risk that the patient will suffer brain damage. Some resuscitation attempts may be traumatic meaning that death occurs in a manner the patient and people close to the patient would not have wished. Where there is a chance of a good or reasonable quality of life being gained, however, most patients willingly risk some disadvantage. A competent patient is the best judge of what represents an acceptable level of burden or risk for him or herself, and where there is a chance of an outcome the patient considers acceptable, many will consider the risk of even significant disadvantage a burden worth taking.

Where the patient is not competent, any previously expressed wishes should form a core part of assessing the benefit to that person. As has been noted above, prolonging life does not always provide a benefit. The courts have confirmed it is lawful to withhold CPR on the basis that it would not confer a benefit upon the patient where consideration has been given to the relevant medical factors and to whether the treatment may provide a reasonable quality of life for the patient. Where patients suffer with such profound disability that they have no or minimal levels of awareness of their own existence and no hope of recovering awareness, or where they suffer severe unmanageable pain or other distress the question arises as to whether initiating treatment to prolong their life would provide a benefit to them. In assessing the benefits that would arise from prolonging life, it is not only legitimate but ethically appropriate to consider whether cardiopulmonary function is likely to fail repeatedly and whether there are any costs to the patient in terms of pain or distressing side effects.

Consideration of the balance of benefits and burdens in these cases also involves difficult matters of balancing rights under the Human Rights Act. The Act guarantees protection for life (Article 2) but also declares that "no one shall be subjected to torture or to inhuman or degrading treatment or punishment" (Article 3). Clearly, such terminology is intended to cover situations in which human beings are deliberately ill-treated and have severe indignities inflicted upon them. Nevertheless, it should be borne in mind that some people have a profound abhorrence of being kept alive in a state of total dependency or permanent lack of awareness. If patients express such views, health professionals should take note. They should refrain from artificially preserving life where it is clear that the patient would consider the resulting situation to be an "inhuman or degrading" state. The duty to protect life must be balanced with the obligation not to subject the patient to inhuman or degrading treatment.

\section{Responsibility for decision making}

The overall responsibility for decisions about CPR and DNAR orders rests with the consultant or GP in charge of the patient's care. He or she should be prepared always to discuss the decision for an individual patient with other health professionals involved in the patient's care, including, in the consultant's case, the patient's GP. The importance of team work and good communication cannot be over-emphasised.

Where care is shared, for example between hospital and general practice, or between general practice and a residential facility, the doctors involved should discuss the issue with each other, with other members of the health care team and with the patient and people close to the patient as appropriate. While responsibility for achieving agreement is a shared task, one individual should take charge of ensuring that the decision is properly recorded and conveyed to all those who need to know it, including locum staff. Local policies should specify who that should be.

\subsection{Clinical decision making}

Health professionals must use their best judgement bearing in mind the clinical condition of the individual patient. Decisions must be based on reliable, up-to-date clinical guidelines. Local policies should include procedures for ensuring that up-todate clinical information is available.

Decisions must always be made on an individual basis. Blanket policies which deny attempts at resuscitation to groups of patients, for example to all patients in a nursing home or to patients above a certain age, are unethical and probably unlawful under provisions of the Human Rights Act which prohibit discrimination in the enjoyment of convention rights.

\section{Recording and communicating decisions}

Any decision about the provision of attempted CPR must be readily accessible to all health professionals who may need to know it, including hospital staff, GPs, deputising or GP co-operative services, and ambulance staff for patients in the community. The patient's known wishes, and decisions relating to attempting CPR should be communicated between health professionals when a patient is referred or discharged.

The entry in the medical records should clearly document and date the decision and the reasons for it, and should be made by the most senior member of the team available. This person should ensure that the decision is communicated effectively to other relevant health professionals in both primary and secondary care. In hospital, the consultant in charge of care will usually lead this process and may delegate the task of disseminating information to another member of the health care team. Where the GP takes the professional lead, he or she has responsibility for these tasks.

The decision should be recorded in the nursing notes by the primary nurse or the most senior member of the nursing team whose responsibility it is to inform other members of the nursing team. 
316 Clinical ethics: Decisions Relating to Cardiopulmonary Resuscitation: a joint statement from the British Medical Association, the Resuscitation Council (UK) and the Royal College of Nursing

Local protocols for information-sharing between health professionals may facilitate communication. Detailed guidance is not provided here since appropriate mechanisms will depend on the type of facilities involved and existing local protocols for communication.

Communication of decisions to the patient and people close to the patient is also a part of this process. Patients who want to be involved in decision making will be aware what decision has been reached and should be told how this will be communicated to the health care team. Health professionals should remember that patients are legally entitled to see and have a copy of their health records. Unless the patient refuses, decisions should also be communicated to the patient's family and others close to the patient. The usual rules of confidentiality apply.

\section{Effects on others}

It is arguable that the rights of people other than the patient, and the potential burdens upon them, are legitimate factors to weigh in the balance of benefits and burdens. Repeated attempts at CPR which provide only short-lived clinical success can be emotionally very difficult for people close to the patient. Repeated failures at resuscitation can lower the morale and enthusiasm of the health care team, and attempting to resuscitate a patient for whom there is no chance of success may mean the staff and equipment are not available to attempt to resuscitate a patient for whom there is a better chance of success. Health professionals should look for ways to minimise these burdens whilst still ensuring that the patient's needs are the paramount consideration. Support for people close to the patient and for health professionals is essential.

\section{Disagreement}

Consensus amongst all those involved in the decision is the aim. Where no advance decision can be reached, a note should be made of the reasons. Documenting discussions and factors to consider can be helpful in the event of cardiopulmonary arrest where no firm advance decision has been made. The health care team should be prepared to attempt CPR unless other factors intervene (such as unavoidable delay in starting the procedure, or if there has been severe deterioration in the patient's condition since the discussion).

Where the clinical decision is seriously challenged and agreement cannot be reached, some form of legal review may be necessary. Legal advice should be sought and local policies may specify how this can be done promptly.

\section{Other treatment}

A decision not to attempt resuscitation applies only to CPR. It should be made clear to the patient, people close to the patient and members of the health care team that it does not imply "non-treatment" and that all other treatment and care that are appropriate for the patient will continue to be considered and offered. To avoid all confusion, the expression "not for attempted cardiopulmonary resuscitation" should be used and included in the patient's notes. Local protocols could provide more detailed instructions on how decisions should be recorded. The BMA issues guidance on decisions to withdraw or withhold other medical treatments which have the potential to prolong life.

\section{Review}

Decisions about resuscitation must be reviewed regularly and in the light of changes in the patient's condition and wishes. The frequency of review should be determined by the health professional in charge and will be influenced by the clinical circumstances of the patient. Nevertheless, local policies may include some general safeguards for ensuring review occurs appropriately. It is important to note that patients' abilities to participate in decision making may vary over time.

The decision reached following admission of the patient to hospital should be reviewed by the consultant in charge at the soonest available opportunity.

\section{Standards}

Any attempt at CPR must be competent and in accordance with established clinical guidelines.

Performance of CPR and experience with DNAR orders should be the subject of clinical audit. The National Institute for Clinical Excellence provides information and resources for clinical audit.

\section{Other published guidance}

Joint Working Party between the National Council for Hospice and Specialist Palliative Care Services and the Ethics Committee of the Association for Palliative Medicine of Great Britain and Ireland. Ethical decision-making in palliative care: cardiopulmonary resuscitation (CPR) for people who are terminally ill. London: National Council for Hospice and Palliative Care Services, August 1997 (available at the Council's website: www.hospice-spccouncil.org.uk).

Resuscitation Council (UK). Resuscitation guidelines 2000. London: Resuscitation Council (UK), 2000.

Royal College of Paediatrics and Child Health, Royal College of Obstetricians and Gynaecologists. Resuscitation of babies at birth. London: BMJ Publishing Group, 1997 (under revision). 